\title{
Inversion of COPROD2 Magnetotelluric Data by Use of ABIC Minimization Method
}

\author{
T. UCHIDA \\ Geophysics Department, Geological Survey of Japan, Tsukuba 305, Japan
}

(Received March 15, 1993; Revised July 28, 1993; Accepted September 1, 1993)

\begin{abstract}
A smooth two-dimensional least-squares inversion was applied to the COPROD2 magnetotelluric (MT) data provided by the Geological Survey of Canada. Selection of the optimum smoothness at each iteration was based on a statistical criterion ABIC (Akaike's Bayesian Information Criterion), which was derived from the Bayes statistics and the entropy-maximization theorem. Six frequencies, below $2 \mathrm{~Hz}$, were picked for the inversion. Both TM and TE mode data at 35 sites were used for the weighted inversion in which the weights were calculated from the observation errors listed in the data file. The MT responses and their partial derivatives with respect to model parameters were calculated with a finite-element method. The inverted model basically shows a three-layer earth: conductive, resistive, and conductive from the surface. Two major conductive anomalies are recognized in the resistive layer of $100 \mathrm{~km}$ thickness. One is interpreted as the North American Central Plains (NACP) anomaly, and the other corresponds to the Thompson Belt (TOBE) anomaly.
\end{abstract}

\section{Introduction}

In order to achieve a stable inversion with a least-squares scheme, we usually add a smoothing operation to the inversion matrix. CONSTABLE et al. (1987) introduced an approach to produce the smoothest model which provides a weighted root-mean-squares (rms) misfit of 1. DEGROOTHEDLIN and CONSTABLE (1990) applied it to a two-dimensional (2-D) inversion of magnetotelluric (MT) data. One problem of their method is that they need a good estimate of observation noises prior to the inversion, because the magnitude of the noises provides a guideline of the final tolerable misfit.

A statistical criterion ABIC (Akaike's Bayesian Information Criterion) developed by AKAIKE (1980) can be also used for the selection of the optimum smoothness. When a model which simultaneously minimizes the misfit and the roughness is sought, we can apply the Bayes' rule to the inversion problem: we take the smoothness constraint as prior information. A Bayesian likelihood is then defined by combining two likelihoods: one is for the misfit minimization and the other is for the roughness minimization. We obtain a model which maximizes the Bayesian likelihood, and the optimum smoothness is chosen in this process. The criterion ABIC is used as an index to find the maximum of the Bayesian likelihood. A smaller ABIC indicates a larger likelihood, hence a better model.

Choice of the optimum smoothness is truly objective by adjusting the contribution of the roughness penalty implicitly in relation to the misfit, or the noise contained in the observed data. Convergence is usually attained automatically, regardless of the magnitude of the observation error. UCHIDA (1993) demonstrated the effectiveness of the ABIC minimization method for 2-D inversions of MT data by applying it to synthetic data and CSAMT field data. Here, the method is utilized for a 2-D inversion of the COPROD2 data provided by the Geological Survey of Canada (GSC) (JoNES, 1988, 1993; JonES and CRAVEN, 1990). 


\section{Method}

The 2-D inversion scheme used in this work is based on the linearized least-squares inversion with a smoothness constraint. For this method, we have to minimize a functional $U$,

$$
U=\|\boldsymbol{W} \boldsymbol{d}-\boldsymbol{W} F(\boldsymbol{m})\|^{2}+\alpha^{2}\|\boldsymbol{C} \boldsymbol{m}\|^{2},
$$

where $\boldsymbol{W}$ is a weighting matrix, $\boldsymbol{d}$ is observed data, and $\boldsymbol{m}$ is a model. A non-linear function $F$ works on the model $\boldsymbol{m}$ to produce MT responses. $\alpha$ is a smoothing parameter, and $\boldsymbol{C}$ is a roughening matrix. The first term on the right hand side is the data misfit and the second term is the model roughness. Since $F$ is non-linear, we first have to linearize it about a starting model. Then we modify the model iteratively to minimize $U$, by solving the following equation:

$$
\left\{(\boldsymbol{W} \boldsymbol{A})^{T}(\boldsymbol{W} \boldsymbol{A})+\alpha^{2} \boldsymbol{C}^{T} \boldsymbol{C}\right\} \boldsymbol{m}_{k+1}=(\boldsymbol{W} \boldsymbol{A})^{T}(\boldsymbol{W} \hat{\boldsymbol{d}}),
$$

where $\boldsymbol{m}_{k+1}$ is the model at $(k+1)$-th iteration, $\boldsymbol{A}$ is a Jacobean matrix consisting of partial derivatives of the MT responses with respect to the model parameters,

$$
\hat{d}=\Delta d+A m_{k}
$$

and

$$
\Delta \boldsymbol{d}=\boldsymbol{d}-F\left(\boldsymbol{m}_{k}\right)
$$

The smoothing factor, which is used to trade-off between the contributions of the two terms, should be carefully selected for a stable inversion. Here, a statistical approach, the ABIC minimization method, is used for this purpose.

A criterion ABIC was derived from the Bayes statistics and the entropy-maximization theorem (AKAIKE, 1980). On applying the Bayesian process to the inversion problem, we define a simultaneous probability density function (pdf) of the misfit minimization and the roughness minimization. A Bayesian pdf (or likelihood), $L(\boldsymbol{m} \mid \boldsymbol{d})$, is defined as

$$
L(\boldsymbol{m} \mid \boldsymbol{d})=\int p(\boldsymbol{d} \mid \boldsymbol{m}) \pi(\boldsymbol{m}) d \boldsymbol{m},
$$

where $p(\boldsymbol{d} \mid \boldsymbol{m})$ is a pdf of the misfit minimization and $\pi(\boldsymbol{m})$ is a pdf of the roughness minimization. We now seek a model that maximizes the Bayesian likelihood. This is equivalent to the minimization of $U$. ABIC was derived through a deduction for the entropy-maximization theorem (see details in AKAIKE, 1980, and UCHIDA, 1993):

$$
\mathrm{ABIC}=(-2) \log (\max L(\boldsymbol{m} \mid \boldsymbol{d}))+2 \text { (number of hyper-parameters) }
$$

The smoothing factor is the only hyper-parameter in this case. A smaller ABIC indicates a larger likelihood, hence a better model. The optimum smoothness is obtained in the process of the likelihood maximization. For the derivation of ABIC, we assume that both of the misfit and the roughness follow zero-mean Gaussian process.

Since ABIC is non-linear with respect to the smoothing factor, we have to search for the optimum one which minimizes ABIC at each iteration. Hence, we need to run the forward calculation as many times as the number of the smoothing parameters tested to find the minimum $\mathrm{ABIC}$ at each iteration.

When we start with an initial model of a homogeneous earth, ABIC is usually a large number for the model because the misfit is large. Then, it decreases as the iteration proceeds, and 
finally converges to a certain value. The chosen smoothing factor also generally decreases as the iteration continues, and the model becomes rougher. We can judge the convergence by checking the decrease rate of $\mathrm{ABIC}$ per iteration.

\section{Inversion Results}

\subsection{Inversion with appropriate weights}

The original COPROD2 data distributed by A. G. Jones of GSC was already static-shift corrected (JONES, 1988). The number of the survey sites is 35, each consisting of approximately 40 period data which range from 0.0026 to 1820 sec. Because of the limitation of the computer memory and the CPU speed, all the data can not be used in the inversion at once. Therefore, six periods, $0.667,2.67,10.7,42.7,171$, and $683 \mathrm{sec}$, were picked for the inversion.

Apparent resistivities and phases of both TM and TE modes were used. Noisy data indicated by A. G. Jones were omitted. The number of the data used is 812 . Although the observation errors shown in the data file seemed to be too small, they were used to calculate the weighting matrix $\boldsymbol{W}$. When the observation error was less than $1 \%$, it was forced to be $1 \%$. Fitting between the observed and theoretical apparent resistivities were measured in the natural logarithmic domain. The Jacobean matrix, weights, and block resistivities were also dealt in the logarithmic domain. One percent error for apparent resistivity corresponds to 0.286 degrees error for phase.

The underground medium was divided into 564 rectangular blocks, each being assigned a distinct resistivity. As will be shown in Fig. 1, larger blocks were used at deep. The forward calculation was by the finite-element method, which solves for the total electric or magnetic field of a given 2-D model (RodI, 1976; OGAWA, 1988). The mesh consisted of rectangular elements, 146 elements horizontally and 38 elements vertically. Since the survey line is very long, more than $400 \mathrm{~km}$, the horizontal size of the elements, $3 \mathrm{~km}$, is not small enough as compared with the skin depth of the highest frequency used. The numerical error for a $5 \Omega \cdot \mathrm{m}$ homogeneous halfspace caused by the mesh discretization is less than $1 \%$ for all periods used. However, there may be inaccurate short period responses at sites where the horizontal resistivity change is large in the inverted model. The initial model was a $100 \Omega \cdot \mathrm{m}$ homogeneous earth. A 2-D Laplacian operator was used for the roughening matrix $C$; the operator was weighted according to horizontal and vertical sizes of a block.

Figure $1 \mathrm{a}$ is the resistivity model after twelve iterations. Figure 2 shows the rms misfit and $\mathrm{ABIC}$ as a function of the smoothing factor at each iteration. The parameter modification successfully reduced the misfit and ABIC until the seventh iteration. After that, however, the solution of Eq. (2) did not provide a smaller ABIC than the previous model, and the modification fell into an oscillating feature. The oscillation was mainly due to the data at sites 32 and 33 , which produced large parameter modification for the blocks beneath the sites. This is possibly because of the breakdown of the linearization assumption for the function $F$. Therefore, a damping operation, similar to the Marquardt method, was added to Eq. (2) after the eighth iteration. The most suitable damping was also decided by the ABIC minimization. Then, a better convergence was attained after twelve iterations.

The weighted rms misfit at the twelfth iteration is 2.6 , although its expected value is around 1 if the noise is Gaussian. This rms misfit corresponds to a relative misfit of approximately $12 \%$. One possible reason for the large rms misfit could be that there is a three-dimensional structure for which the 2-D assumption is invalid. Another reason could be that the observation errors are much larger than those given. If we assume that the earth is two-dimensional and the noise is Gaussian, the true observation error of each data is generally 2.6 times as large as the listed value. The resultant model by the ABIC minimization method is independent of these underestimated errors. Also, a relative contribution of each datum is preserved. 
(a) COPROD2 1\%-min ABIC iteration=12 $\mathrm{rms}=2.59$

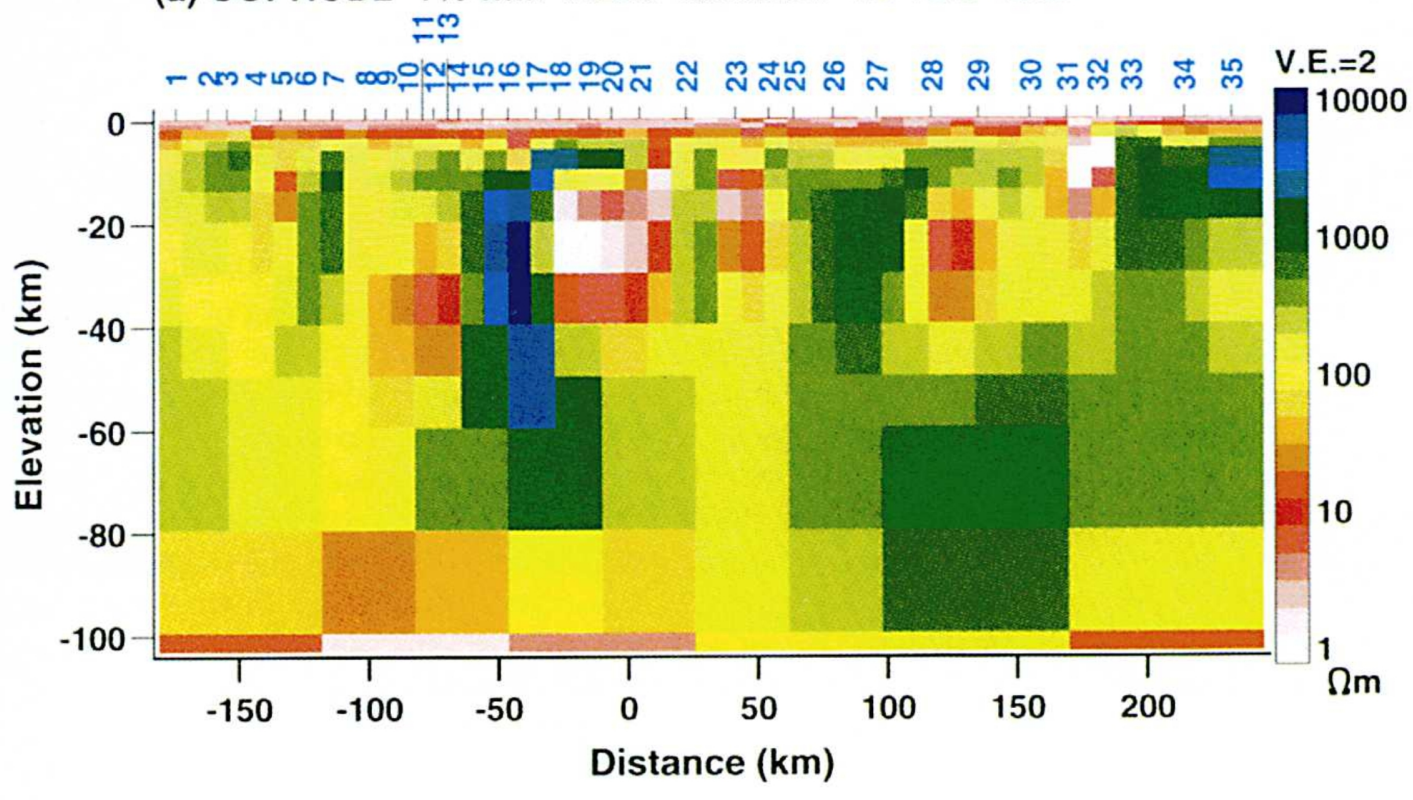

(b) COPROD2 10\%-min ABIC iteration $=14 \mathrm{rms}=0.84$

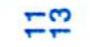

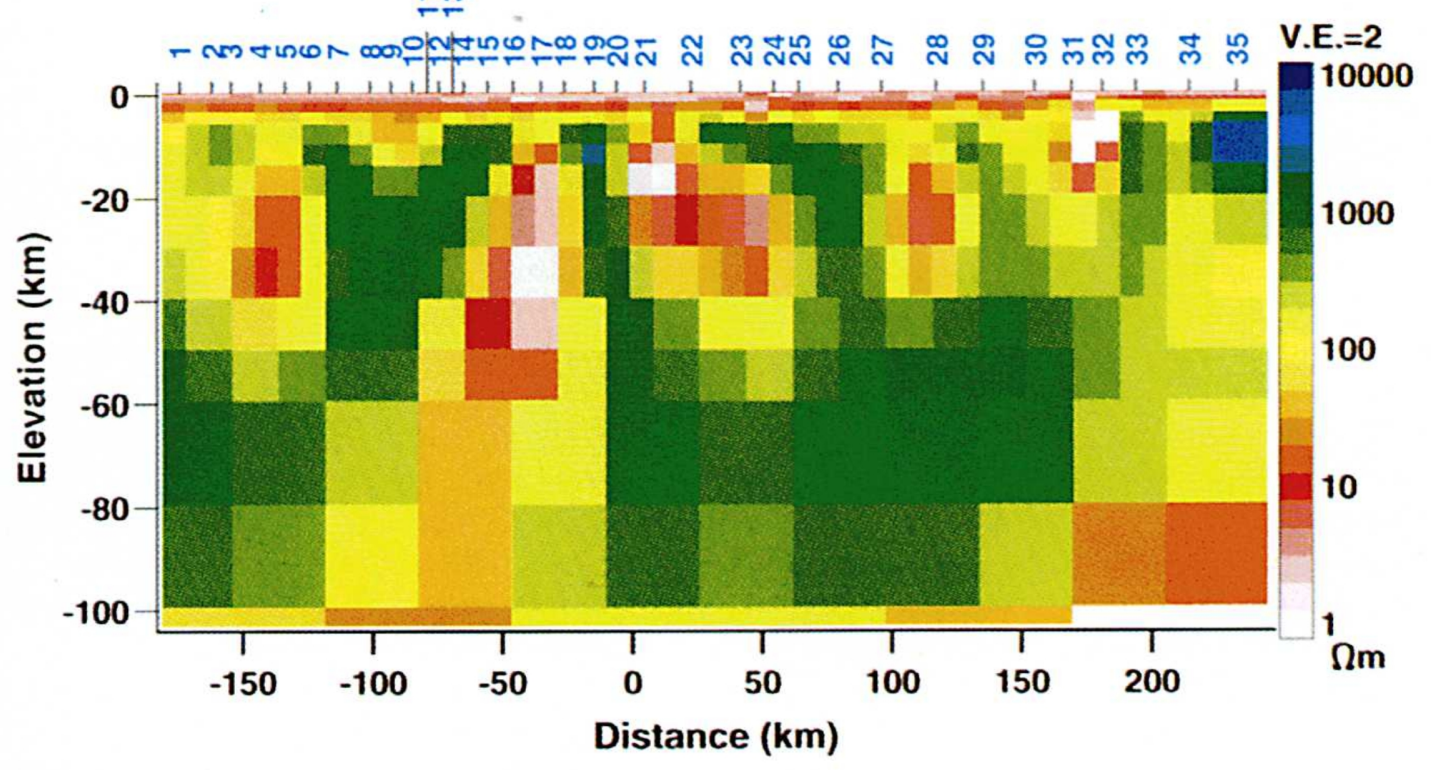

Fig. 1. A 2-D model of smooth inversion of the COPROD2 TE and TM data at six periods: $0.667,2.67,10.7$, $42.7,171$, and $683 \mathrm{sec}$. Minimum noises of (a) $1 \%$ and (b) $10 \%$ were assumed. The number of the data used is 812 , while there are 564 blocks in the model. The initial guess is a $100 \Omega \cdot \mathrm{m}$ homogeneous earth. Vertical exaggeration is 2 . 'rms' stands for a weighted root-mean-squares misfit. 
(a)



(c)

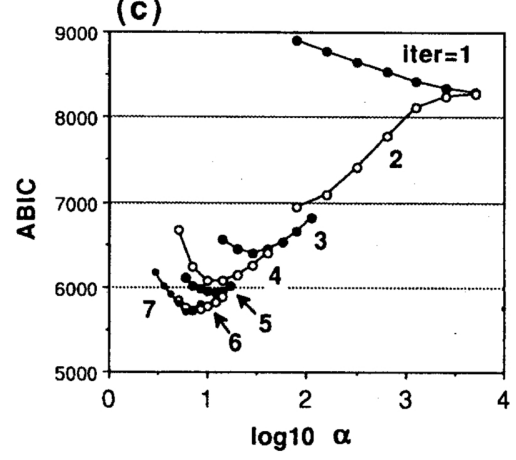

(b)
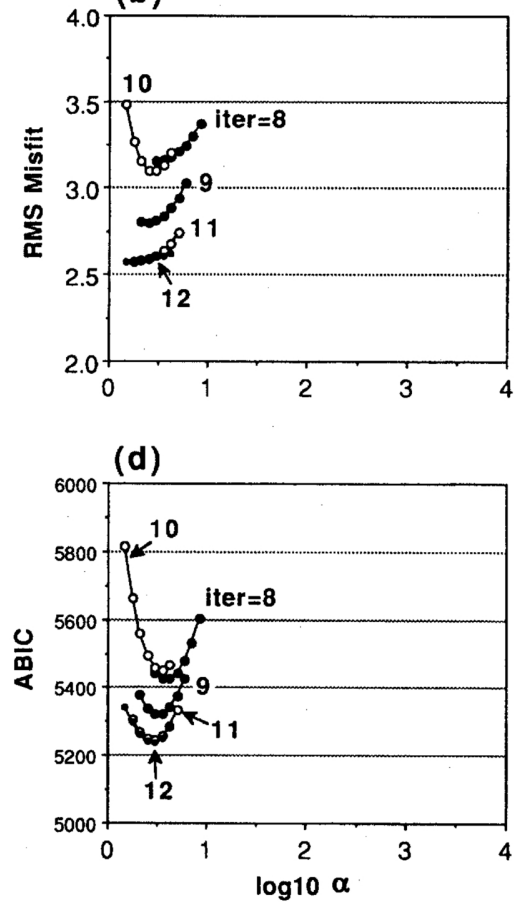

Fig. 2. Weighted rms misfit and ABIC as a function of the smoothing factor at each iteration for the one-percent minimum case (Fig. 1a). (a) and (c) are for up to the seventh iteration, while (b) and (d) are for eight through twelve iterations. The vertical axes are blown up for figures (b) and (d). The optimum smoothing factor was chosen at the minimum ABIC at each iteration.

Apparent resistivities and phases, both observed and modelled, are shown in Fig. 3. Fitting between the observed and calculated data are visually very good on the sounding curves, although they still have an average misfit of $12 \%$. Figure 4 shows the weighted residuals of the responses for the model in Fig. 1a. A minimum observation error of $1 \%$ is assumed. Most of relatively large disagreements on the sounding curves (Fig. 3) are due to large data errors of the corresponding data.

\subsection{Effect of noise estimation}

One more inversion was conducted by assigning different weights with an assumption of tenpercent minimum noise. A noise less than $10 \%$ was forced to be $10 \%$. Since the average of the observation noises is approximately $6 \%$, this is similar to an equally weighted inversion. The noise level of the data at periods of 10-100 sec. is generally much less than $10 \%$, while it often reaches more than $10 \%$ for the data at around 1 and 1000 sec. The mid-period data correspond to a depth of the transition from the conductive first layer to the resistive second layer. Relative contribution of those data becomes smaller for the inversion of ten-percent minimum noise than the case of one-percent minimum noise.

The inversion was performed in the same manner as described above. The damping operation was applied after ten iterations, and convergence was attained at the fourteenth iteration. The model obtained is shown in Fig. $1 \mathrm{~b}$; the final $\mathrm{rms}$ misfit is 0.84 , and a relative misfit is approximately $10 \%$. Roughness defined by $\|\boldsymbol{C m}\|^{2}$ is 134 , while it is 213 for the case of onepercent minimum noise (Fig. 1a). The general feature of the model is very similar to that of Fig. 1a, except for the structure of the NACP anomaly, which is located beneath the sites 15 
(a) TE

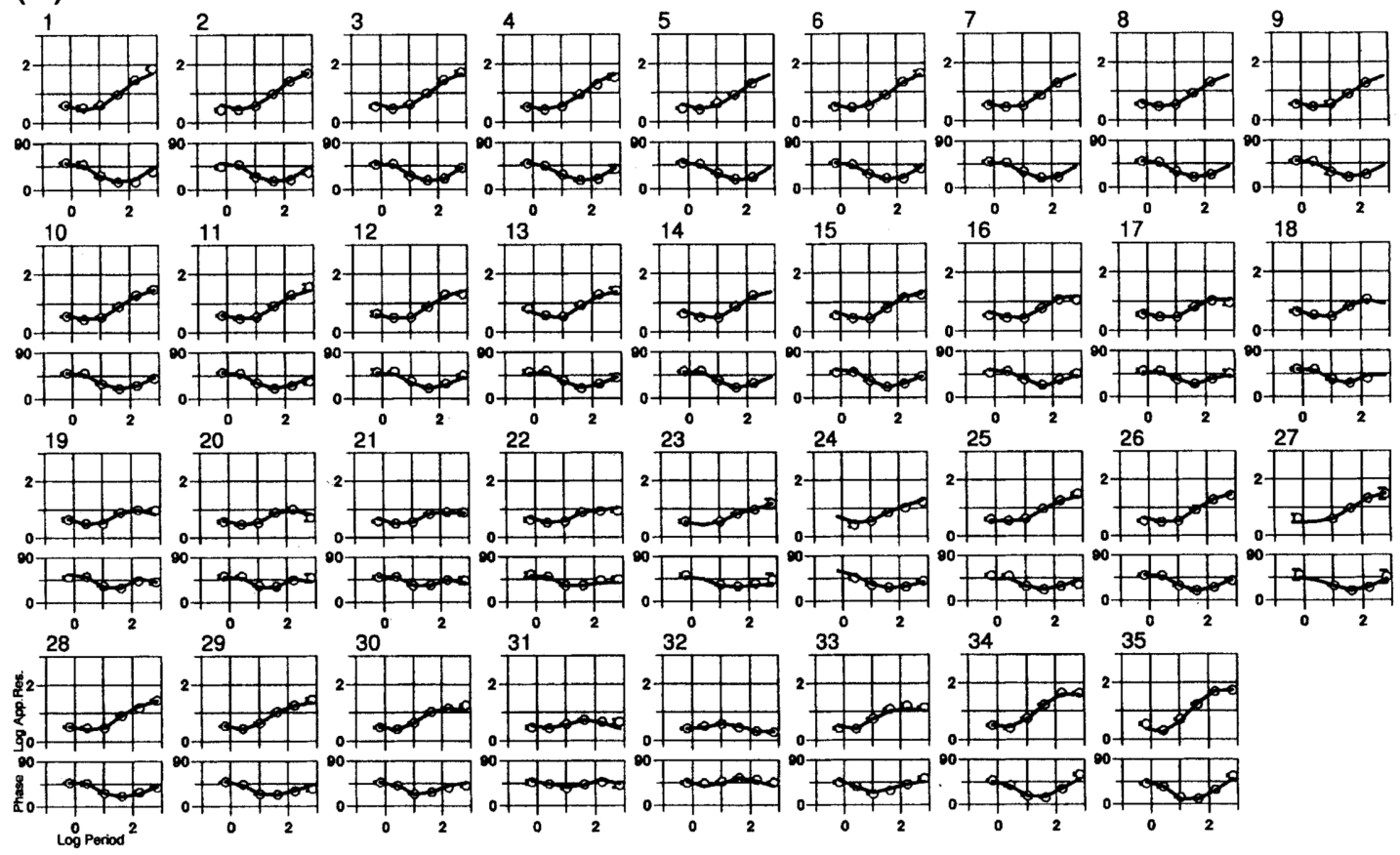

(b) TM

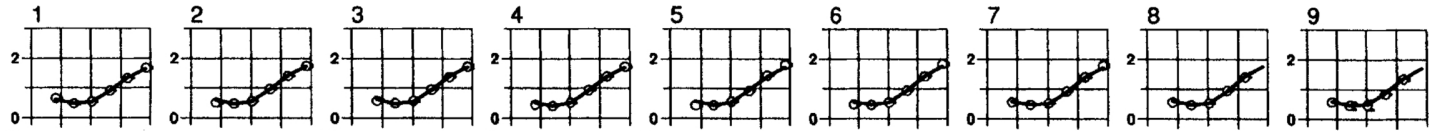

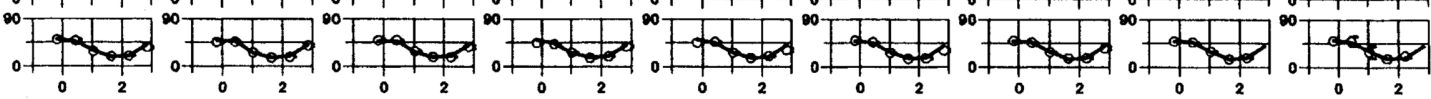

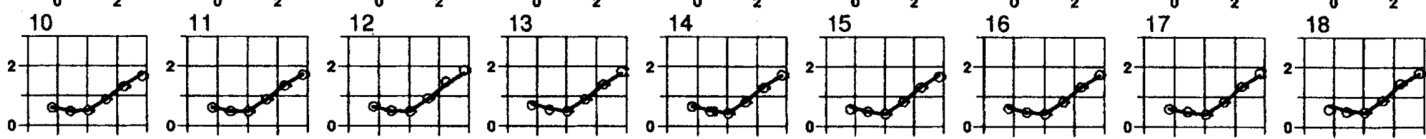

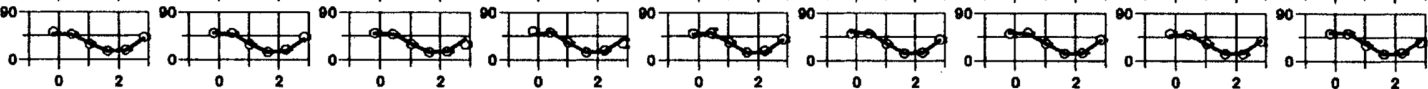
华

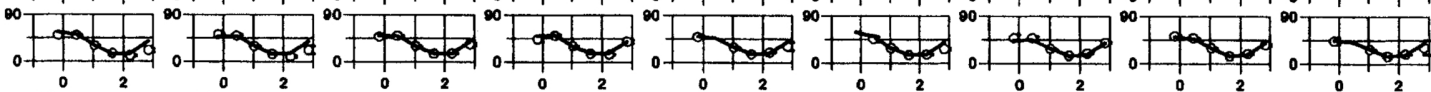

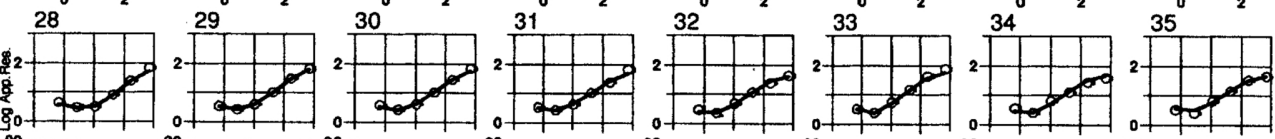



Fig. 3. Observed apparent resistivities and phases (open circles) and calculated ones from the model shown in Fig. 1a (solid lines) for (a) TE mode and (b) TM mode. Apparent resistivity in a $\log _{10} \Omega \cdot \mathrm{m}$ scale, phase in degree, and period in $\log _{10}$ second are shown for each survey site. 


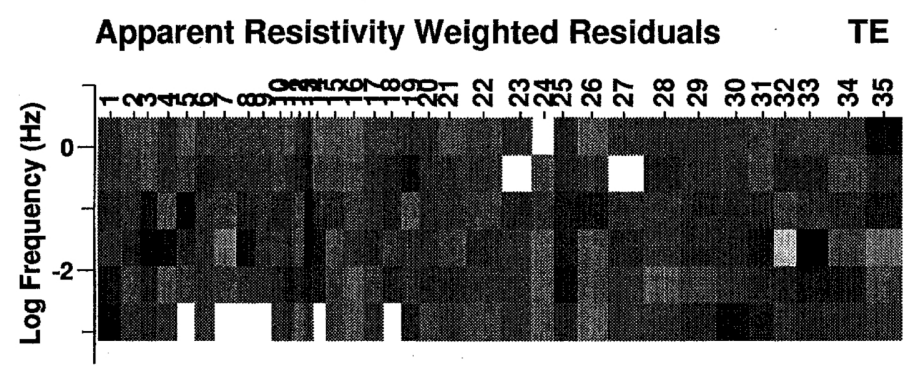

\section{Phase Weighted Residuals}

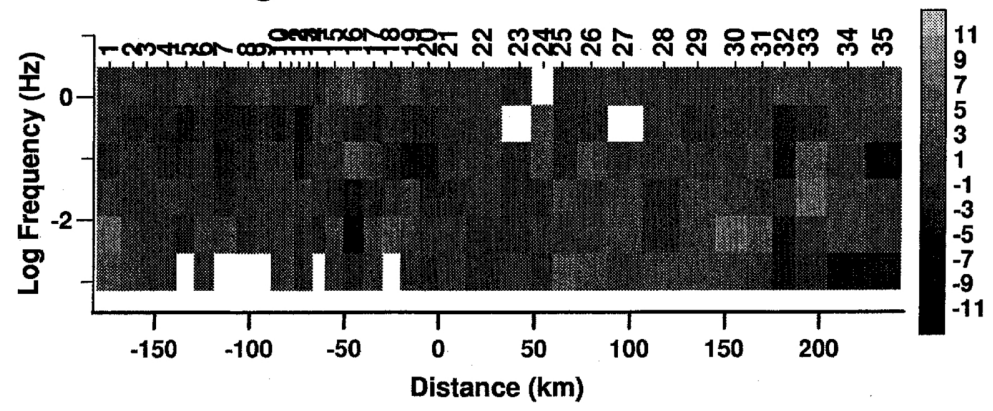

(a)

\section{Apparent Resistivity Weighted Residuals}



\section{Phase Weighted Residuals}



(b)

Fig. 4. Weighted residuals between the observed and the theoretical values for (a) TE mode and (b) TM mode. A minimum noise of $1 \%$ is assumed. 
to 23. Although both models show two conductors at a depth of $15-40 \mathrm{~km}$, their locations are different between these models. This suggests that the resultant model is very sensitive to the data quality, and that a careful estimation of the noise is essential.

The inversion by the ten-percent minimum noise provides a smoother model which also has a smaller relative misfit than the one by the one-percent minimum noise. Yet, since the noise information of the original data is better preserved in the model of Fig. 1a, I regard it as a more suitable model.

\subsection{Interpretation}

We can recognize a surficial resistive layer of a few tens of $\Omega \cdot \mathrm{m}$ from the original data at around $100 \mathrm{~Hz}$. However, they were omitted from the inversion. The inverted model basically consists of three layers (Fig. 1a): conductive first layer of $2-5 \Omega \cdot \mathrm{m}$, resistive second layer of 100 $1000 \Omega \cdot \mathrm{m}$, and conductive third layer of $1-10 \Omega \cdot \mathrm{m}$. The first conductive layer is a few kilometer thick, and the third layer is approximately 100 kilometer deep. Within the resistive layer, five conductive anomalies are recognized: they are beneath sites 9-14, 18-21, 23-24, 28-29, and 31-32. The most significant one, beneath the sites 18-21, corresponds to the NACP anomaly, together with the conductor at the sites 23-24. Small but very conductive and shallow one at the sites 28-29 is the TOBE anomaly.

Both of the models in Fig. 1 indicate two conductors for the NACP anomaly. However, they may be due to small distortions of the corresponding data. Because the apparent resistivity pseudo-section of the TE mode indicates only a single large conductor for the NACP anomaly and the TM data hardly detect the anomaly, the split conductors are not necessarily required to explain the data.

\section{Conclusions}

A two-dimensional inversion with the ABIC-minimization method was conducted for the COPROD2 data. Although the convergence was not so stable as compared with the cases of synthetic data or CSAMT field data of good quality, the result is reasonable to explain the field data and general features of the underground structure. The inverted model greatly depends on how we set the weights from the noise information. The final model indicates a three-layer earth: conductive, resistive and conductive layers from the surface. Five conductive anomalies are analyzed in the resistive second layer. Two major ones are interpreted as the NACP and TOBE anomalies.

The author is grateful for Y. Ogawa and Y. Mitsuhata of Geological Survey of Japan for providing useful comments on this work. He also wishes to thank P. J. Savage of PanCanadian for making the raw data available and A. G. Jones of GSC for providing the static shift corrected COPROD2 data.

\section{REFERENCES}

Akaike, T., Likelihood and Bayes procedure, in Bayesian Statistics, edited by J. M. Bernardo, M. H. deGroot, D. V. Lindley, and A. F. Smith, pp. 143-166, University Press, Valencia, Spain, 1980.

Constable, S. C., R. L. Parker, and G. Constable, Occam's inversion: a practical algorithm for generating smooth models from electromagnetic sounding data, Geophysics, 52, 289-300, 1987.

DEGroot-Hedlin, C. and S. C. Constable, Occam's inversion to generate smooth, two-dimensional models from magnetotelluric data, Geophysics, 55, 1613-1624, 1990.

Jones, A. G., Static shift of magnetotelluric data and its removal in a sedimentary basin environment, Geophysics, 53, 967-978, 1988.

JONES, A. G., The COPROD2 dataset: Tectonic setting, recorded MT data and comparison of models, J. Geomag. Geoelectr., this issue, 933-955, 1993. 
Jones, A. G. and J. A. Craven, The North American Central Plains conductivity anomaly and its correction with gravity, magnetic, seismic, and heat flow data in Saskatchewan, Canada, Phys. Earth Planet. Inter., 60, 169-194, 1990.

OGAWA, Y., Fortran program codes for two-dimensional magnetotelluric forward and inverse analysis, Geol. Surv. Japan Open-File Report, No. 59, 96 pp., 1988 (in Japanese).

RodI, W. L., A technique for improving the accuracy of finite element solutions for magnetotelluric data, Geophys. J. R. astr. Soc., 44, 483-506, 1976.

UCHIDA, T., Smooth 2-D inversion for magnetotelluric data based on statistical criterion ABIC, J. Geomag. Geoelectr., this issue, 841-858, 1993. 